\title{
Validation of reported physical activity for cholesterol control using two different physical activity instruments
}

This article was published in the following Dove Press journal:

Vascular Health and Risk Management

7 August 2009

Number of times this article has been viewed

\author{
Amy Z Fan' \\ Sandra A Ham² \\ Shravani Reddy Muppidi ${ }^{3}$ \\ Ali $\mathrm{H} \mathrm{Mokdad}^{4}$ \\ 'Behavioral Surveillance Branch, \\ Division of Adult and Community \\ Health, National Center for Chronic \\ Disease Prevention and Health \\ Promotion; ${ }^{2}$ Physical Activity and \\ Health Branch, Division of Nutrition, \\ Physical Activity, and Obesity, \\ National Center for Chronic \\ Disease Prevention and Health \\ Promotion, Centers for Disease \\ Control and Prevention, Atlanta, \\ GA, USA; ${ }^{3}$ College of Public Health, \\ University of Georgia, Athens, \\ GA, USA; ${ }^{4}$ Institute for Health \\ Metrics and Evaluation, University \\ of Washington, Seattle, WA, USA
}

Correspondence: Amy Z Fan

Behavioral Surveillance Branch, Division of Adult and Community Health, NCCDPHP, Centers for Disease Control and Prevention, 4770 Buford Highway, NE, MS K-66, Atlanta, GA 3034I, USA

Tel + I 7704885327

Fax +I 7704888150

Email afan@cdc.gov

\begin{abstract}
The National Cholesterol Education Program recommends increasing physical activity to improve cholesterol levels and overall cardiovascular health. We examined whether US adults who reported increasing their physical activity to control or lower blood cholesterol following physician's advice or on their own efforts had higher levels of physical activity than those who reported that they did not. We used data from the National Health and Nutrition Examination Survey 2003-2004, which implemented two physical activity assessment instruments. The physical activity questionnaire (PAQ) assessed self-reported frequency, intensity, and duration of leisure-time, household, and transportation-related physical activity in the past month. Physical movement was objectively monitored using a waist accelerometer that assessed minute-by-minute intensity (counts of movement/minute) during waking time over a 7-day period. We adjusted our analysis for age, gender, race/ ethnicity, educational attainment, and body mass index. Participants who reported increasing physical activity to control blood cholesterol had more PAQ-assessed physical activity and more accelerometer-assessed active days per week compared to those who did not. However, there were no significant differences in cholesterol levels between comparison groups. These findings suggest that self-report of exercising more to control or lower cholesterol levels among US adults might be valid.
\end{abstract}

Keywords: accelerometer, cardiovascular diseases, hypercholesterolemia, physical activity, risk reduction behavior

Epidemiologic evidence has shown that elevated low-density lipoprotein cholesterol (LDL-C) is a major cause of coronary heart disease (CHD). ${ }^{1,2}$ Strong evidence also establishes the inverse relationship of high-density lipoprotein cholesterol (HDL-C) with cardiovascular morbidity and mortality. ${ }^{3-6} \mathrm{HDL}$ is protective against cardiovascular disease by inhibiting the oxidative modification of LDL. ${ }^{7}$ The cardio-protective action of physical activity can be attributed to the elevation of HDL, ${ }^{8-14}$ and decrease in LDL and triglyceride levels. ${ }^{8-10,12,13}$ Low to moderate levels of physical activity are sufficient to bring about changes in the lipid profile of hypercholesterolemic patients. ${ }^{8,9,11,13,14}$ The National Cholesterol Education Program (NCEP) also recommended an increase in physical activity for hypercholesterolemic patients as a method of primary prevention of CHD. ${ }^{1}$ The US Department of Health and Human Services recommends 150 minutes of moderate-intensity physical activity per week, or 75 minutes of vigorous-intensity activity per week, or an equivalent combination of moderate- and vigorous-intensity physical activity (MVPA). ${ }^{15}$ Activity can 
be performed in bouts of 10 minutes or more. However, a large proportion of the population does not meet these recommendations. ${ }^{16,17}$

Physicians play an important role in promoting physical activity. Patients are more likely to increase their physical activity levels if their physician had advised such measures. ${ }^{18-23}$ Although a number of studies have been conducted to examine the influence of physician advice on physical activity in normal adults, no studies have been conducted among hypercholesterolemic adults. In this study we examined whether hypercholesterolemic participants who reported increasing their physical activity (compliant group) indeed had higher levels of physical activity than those who reported that they did not exercise more (noncompliant group) with two physical activity assessment instruments - namely, the National Health and Nutrition Examination Survey (NHANES) physical activity questionnaire (PAQ) and the accelerometer. We were interested in validating the self-reported PAQ in the NHANES with accelerometer data in samples defined based on concern about hypercholesterolemia. In addition, we were interested in understanding the type, frequency, intensity, and duration of activities that each group performed. We also did similar comparisons among participants who were not told they had high cholesterol but still increased physical activity to control cholesterol. Finally, we assessed whether the groups that reported increased physical activity possessed better lipid profiles than the low activity groups.

\section{Materials and methods}

The NHANES is a household interview and examination survey among the civilian, noninstitutionalized US population selected using a complex, multi-stage probability design. In 2003-2004, data were collected for 10,122 persons of all ages. The participants who were aged 18 years and older, not pregnant, and who answered the cholesterol check-up questions and the PAQ were included in our analysis $(n=4,637)$. The study participants were initially interviewed at their residence to obtain data for demographic and family characteristics. The PAQ section was administered in the household interview. The PAQ section includes an extensive array of questions related to daily activities, leisure time activities, and sedentary activities at home. Participants were invited to a Mobile Examination Center (MEC) for a health examination and an in-person survey interview of health behaviors including cholesterol checkup, physician counseling to increase physical activity to lower blood cholesterol, and physical activity behaviors.
Ambulatory participants were asked to wear a uniaxial accelerometer (ActiGraph model 7164; ActiGraph, LLC, Pensacola, FL) for seven consecutive days during waking hours to monitor their physical movement. Accelerometers are small, electronic devices that record acceleration of change of bodily movement and provide an objective estimate of duration and intensity of locomotion. ${ }^{24}$ Participants were asked to return the accelerometers in an envelope with prepaid postage following the monitoring period. The details on physical activity measures derived from PAQ and accelerometer are shown in Appendix 1.

Blood specimens were collected at the MECs. General information on specimen collection and quality control for laboratory data is available at http://www.cdc.gov/ nchs/data/nhanes/nhanes_03_04/lab_c_generaldoc.pdf. The lab methodology for assessment of total cholesterol and HDL-cholesterol were available at http://www.cdc.gov/nchs/ data/nhanes/nhanes_03_04/113_c.pdf. The lab methodology for the assessment of triglycerides is available at http://www. cdc.gov/nchs/data/nhanes/nhanes_03_04/113am_c.pdf. LDL- cholesterol is calculated from measured values of total cholesterol, triglycerides, and HDL-cholesterol according to the Friedewald calculation.

About 4,637 eligible participants were asked whether they had their blood cholesterol checked and whether they were told their cholesterol was high. The participants who were told they had high cholesterol $(n=1,441)$ were then asked whether they were told by health professionals to exercise more to control their cholesterol and whether they followed the advice. Among those who were never told they had high blood cholesterol $(n=3196)$, the following questions were asked: "Have you made any major changes on your own to lower your blood cholesterol? Specifically, have you increased your physical activity or exercise in order to lower your blood cholesterol?" We analyzed data from four groups: hypercholesterolemic adults who received advice and self-reported that they were $(n=655)$ or were not compliant $(\mathrm{n}=293)$, and adults who were never told to have hypercholesterolemia increased $(n=552)$ or did not increase $(n=2,644)$ physical activity on their own to lower their cholesterol levels. The step-by-step sample distribution is shown in Figure 1.

\section{Covariates}

Age and sex were self-explanatory. Four categories of race/ ethnicity were created: non-Hispanic white, non-Hispanic black, Mexican American, and others. Years of education is a three-category variable that groups survey participants into 


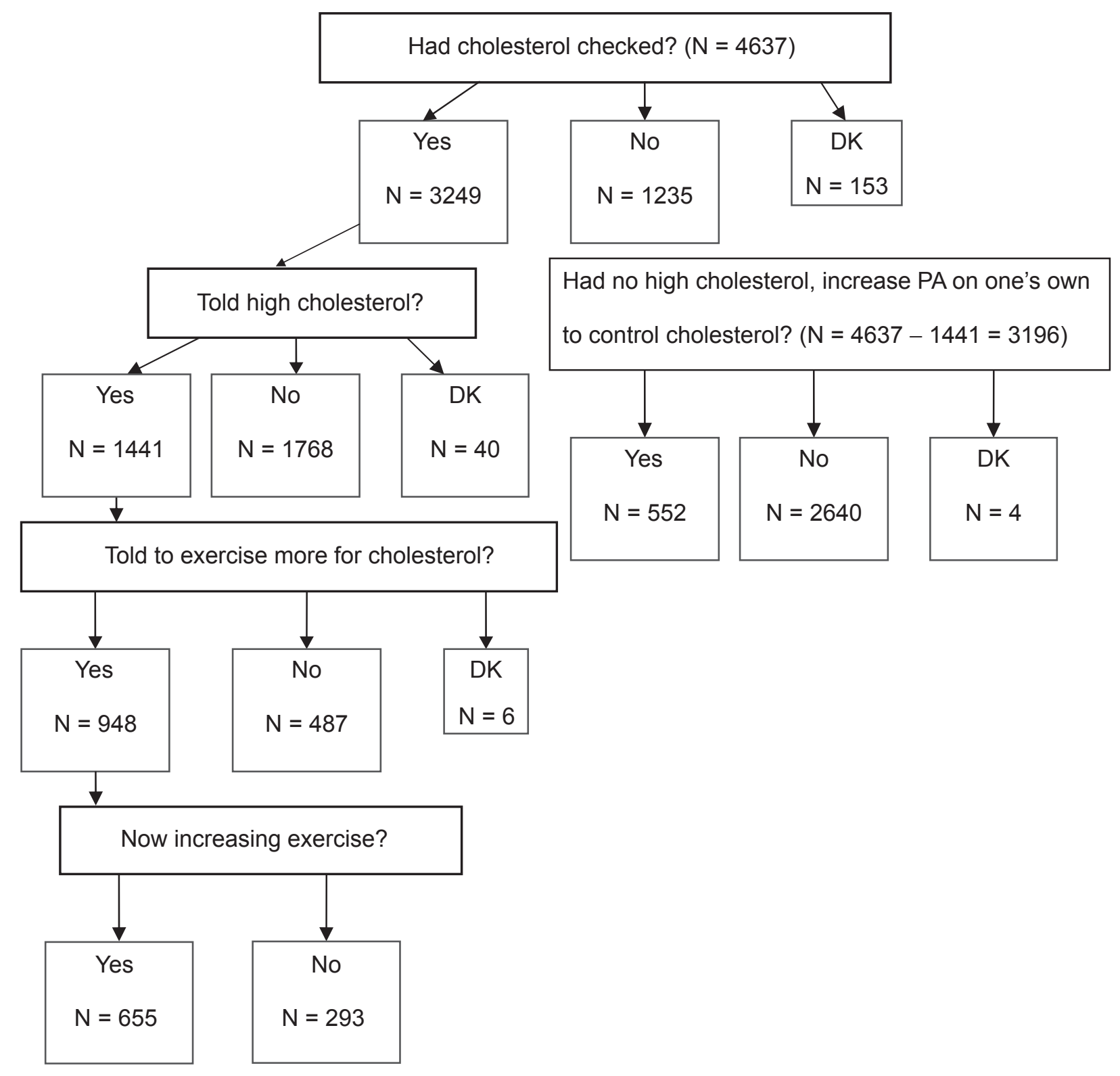

Figure I Sample distribution. National Health and Nutrition Examination Survey 2003-2004.

Abbreviation: DK, don't know.

one of three educational attainment groups: 1) less than high school education attainment, 2) high school graduate (has a high school diploma or high school equivalency diploma such as a General Educational Development [GED]), or 3) has more than a high school education. Body mass index (BMI) was calculated as weight in kilograms divided by the square of height in meters.

\section{Statistical analysis}

The analysis was performed using SAS-callable SUDAAN (Release 9.0.1, Research Triangle Institute, Research Triangle Park, NC, 2007) to account for the complex sampling design of NHANES. We used generalized linear modeling to compare physical activity levels between groups for measures of physical activity based on different domains (ie, exercise, sports, recreation, household physical activity, transportation physical activity, all physical activity), types of physical activity (ie, cardio, strengthening, flexibility), and physical activity based on intensity (ie, moderateintensity physical activity, vigorous-intensity physical activity). The least-square means of continuous questionnairederived variables were obtained with adjustment for age, gender, race/ethnicity, educational attainment, and BMI category. The least-square means of continuous accelerometer-derived 
variables were obtained with adjustment for age, gender, race/ethnicity, educational attainment, BMI category, and days of valid accelerometer wear. All analyses were weighted. For the latter comparisons, we used sample weights, which reflects the additional "nonresponse" related to not having complete accelerometer data. Serum triglyceride and LDL cholesterol were available only for those who had fasted at least eight hours before their laboratory examination in the morning. Therefore, separate weight for morning fasting sample was used for these variables. Some variables were log-transformed before the analysis because of right skewness and transformed back to original scales for comparison. $P$ values are two-sided.

\section{Results}

Our study population (nonpregnant, 18 years or older) consisted of $74 \%$ non-Hispanic whites, $11 \%$ non-Hispanic blacks, 8\% Mexican Americans, and 7\% others. The average age was 46 years. About $48 \%$ were male and 55\% attained high school or higher education. About $35 \%$ of this population were overweight and 32\% were obese. Among this population, about $70.8 \%$ (95\% confidence interval [CI]: $67.6 \%, 73.8 \%$ ) had their cholesterol checked. Among the adults checked, $42.2 \%$ (95\% CI: $39.7 \%$, 44.7\%) were told they have high blood cholesterol. Among these adults with high blood cholesterol, about 67.0\% (95\% CI: 62.7\%, 71.0\%) were told to increase physical activity in order to lower blood cholesterol. Among the adults given advice, $68.1 \%$ (95\% CI: 64.4\%, 71.7\%) reported having followed the advice. Among those who had no high blood cholesterol and never received such advice, only $15.3 \%$ (95\% CI: $13.2 \%, 17.8 \%)$ increased physical activity on their own.

Among the hypercholesterolemic adults who were advised by their physician to increase physical activity levels, the compliant adults had a greater number of sessions and more total MET-minutes of all physical activity per month compared to the noncompliant adults $(P<0.0001$; Table 1$)$. Based on the intensity of physical activity, the compliant adults had greater numbers of sessions and MET-minutes of moderate- and vigorous-intensity physical activity per month compared to the noncompliant adults $(P<0.0001)$. From the accelerometer data during wear periods from all valid days, the compliant adults also had greater mean intensity counts per minute $(P=0.0006)$, mean counts per minute of most intense 10 minutes per week $(P=0.001)$, and mean duration (minutes) of moderate- and vigorous-activity bouts (minimum one minute bouts) per day from all valid days $(P=0.0008)$. Compliant adults also had higher mean duration (minutes) of moderate- and vigorous-activity bouts (eight out of 10 minute bouts) per day from all valid days $(P=0.0002)$ and more days per week of greater than or equal to 10 minutes of MVPA bouts (eight out of 10 minutes) from all valid days $(P<0.0001)$. However, there was no difference in adherence to physical activity recommendations based on Bayesian probability estimates.

Among nonhypercholesterolemic adults who were never told to increase physical activity to lower cholesterol, adults who increased their physical activity on their own had a greater number of sessions and total MET-minutes of all physical activity per month compared to the adults who did not increase physical activity on their own $(P<0.001$; Table 2). They also had more MET-minutes of exercise $(P<0.0001)$ per month. Based on the type of physical activity, the adults that increased physical activity on their own had a larger number of sessions and more total METminutes of cardio and strengthening exercises per month compared to those who did not increase physical activity. Based on the intensity of physical activity, the adults who increased physical activity on their own had more sessions of moderate- $(P=0.0003)$ and vigorous- $(P<0.0001)$ intensity physical activity per month and more MET-minutes of vigorous-intensity physical activity $(P<0.0001)$ than those who did not increase physical activity. The accelerometer data showed that the adults who increased physical activity on their own had greater mean counts per minute of most intense 10 minutes per week of physical activity $(P=0.044)$, mean duration (minutes) of moderate- and vigorous-activity bouts (minimum one minute bouts) per day from all valid days $(P=0.03)$, mean duration (minutes) of moderate- and vigorous-activity bouts (eight out of 10 minute bouts) per day from all valid days $(P=0.046)$, and more days per week of greater than or equal to 10 min of MVPA bouts (eight out of 10 minutes) from all valid days $(P=0.026)$. However, there was no difference in adherence to recommendations for physical activity based on Bayesian probability estimates.

The sample used in the accelerometer data analysis had at least one valid day of accelerometer data. Alternatively, we restricted our analysis to the sample that had four or more valid days of accelerometer data and obtained similar results.

No significant difference in blood lipid profiles (total cholesterol, LDL-C, HDL-C, triglycerides) were found between hypercholesterolemic adults who increased physical activity levels and those who did not and between nonhypercholesterolemic adults who increased physical activity levels on their own and those who did not (Tables 1 and 2). The analysis was adjusted for age, gender, race/ethnicity, educational attainment, and BMI category. Controlling for 
Table I Physical activity (PA) measures (adjusted mean, [95\% CI]) assessed by PAQ and by accelerometer and lipids profile based on whether the hypercholesterolemic participants followed the physician's advice to increase PA levels

\begin{tabular}{|c|c|c|c|}
\hline Questionnaire-derived measures & $\begin{array}{l}\text { Compliant } \\
(n=655)\end{array}$ & $\begin{array}{l}\text { Noncompliant* } \\
(n=293)\end{array}$ & $\begin{array}{l}P \text { for } \\
\text { difference }\end{array}$ \\
\hline \multicolumn{4}{|l|}{$\begin{array}{l}\text { I) PA based on different domains } \\
\text { (number of sessions per month): }\end{array}$} \\
\hline Exercise & $19.7(16.6-22.8)$ & $4.7(2.9-6.5)$ & $<0.0001$ \\
\hline Sports & $0.9(0.5-1.3)$ & $0.4(0.2-0.6)$ & 0.13 \\
\hline Recreation & $1.9(1.3-2.5)$ & $0.3(0.1-0.5)$ & $<0.0000 \mathrm{I}$ \\
\hline Household PA & $7.7(6.5-8.9)$ & $5.8(4.8-6.8)$ & 0.02 \\
\hline Transportation PA & $2.5(1.3-3.7)$ & $2.3(0.3-4.3)$ & 0.8 \\
\hline All PA & $32.7(29.2-36.2)$ & $13.5(10.6-16.4)$ & $<0.0001$ \\
\hline $\begin{array}{l}\text { 2) PA based on different domains } \\
\text { (total MET-minutes): }\end{array}$ & & & \\
\hline Exercise & $2988(2428-3548)$ & $609(344-873)$ & $<0.0001$ \\
\hline Sports & $415(187-643)$ & $207(82-332)$ & 0.2 \\
\hline Recreation & $674(392-955)$ & $142(12-263)$ & 0.0070 \\
\hline Household PA & $3470(2727-4212)$ & $2694(1902-3486)$ & 0.22 \\
\hline Transportation PA & $250(\mid 27-372)$ & $156(26-285)$ & 0.23 \\
\hline All PA & 7796 (7037-8555) & $3808(3 \mid 27-4488)$ & $<0.0001$ \\
\hline \multicolumn{4}{|l|}{ 3) Type of PA: } \\
\hline \multicolumn{4}{|l|}{ Number of sessions per month } \\
\hline Cardio & $29.3(26.2-32.4)$ & $12.8(10-15.5)$ & $<0.0001$ \\
\hline Strengthening & I.04 (0.3-I.8) & $0.3(0.1-0.5)$ & 0.013 \\
\hline Flexibility & $2.3(1.7-2.9)$ & $0.5(0.1-1.0)$ & 0.0005 \\
\hline \multicolumn{4}{|l|}{ Total MET-minutes } \\
\hline Cardio & $7603(6854-8352)$ & $3735(3056-44 I 5)$ & $<0.0001$ \\
\hline Strengthening & $108(49-167)$ & $50(0-116)$ & 0.20 \\
\hline Flexibility & $85(62-109)$ & $23(0-49)$ & 0.0007 \\
\hline \multicolumn{4}{|l|}{ 4) PA based on intensity } \\
\hline \multicolumn{4}{|l|}{ Number of sessions per month } \\
\hline Moderate intensity PA & $24.3(22.0-26.6)$ & $12.2(9.6-14.7)$ & $<0.0001$ \\
\hline Vigorous intensity PA & $5.0(3.6-6.4)$ & $0.5(0-1.1)$ & $<0.0001$ \\
\hline \multicolumn{4}{|l|}{ Total MET-minutes } \\
\hline Moderate intensity PA & $6303(5563-7043)$ & $3534(2819-4250)$ & $<0.0001$ \\
\hline Vigorous intensity PA & $1299(916-1683)$ & $201(I I-39 I)$ & $<0.0001$ \\
\hline Accelerometer-derived measures & $\mathbf{N}=\mathbf{5 5 7}$ & $\mathbf{N}=232$ & \\
\hline $\begin{array}{l}\text { †ean intensity counts/minute on wear } \\
\text { periods from all valid days }\end{array}$ & $264(254-275)$ & $229(216-243)$ & 0.0006 \\
\hline $\begin{array}{l}\text { †Mean counts/minute of most intense } \\
10 \mathrm{~min} / \text { week }\end{array}$ & $24 I 5(2277-2,562)$ & 2079 (1960-2205) & 0.001 \\
\hline $\begin{array}{l}\text { †Mean duration (minutes) of moderate } \\
\text { and vigorous activity bouts (minimum } \\
\text { I minute bouts) per day from all valid days }\end{array}$ & $12.1(10.9-13.4)$ & $9.1(8.0-10.3)$ & 0.0008 \\
\hline $\begin{array}{l}\text { †Mean duration (minutes) of moderate and } \\
\text { vigorous activity bouts ( } 8 \text { out of } 10 \text { minute } \\
\text { bouts) per day from all valid days }\end{array}$ & $1.8(1.5-2.1)$ & $0.8(0.6-1.1)$ & 0.0002 \\
\hline $\begin{array}{l}\text { Days/week } \geq 10 \mathrm{~min} \text { of MVPA bouts } \\
\text { ( } 8 \text { out of } 10 \text { minutes) from all valid days }\end{array}$ & $1.0(0.9-1.2)$ & $0.5(0.4-0.7)$ & $<0.000$ I \\
\hline
\end{tabular}


Table I (Continued)

\begin{tabular}{|c|c|c|c|}
\hline Questionnaire-derived measures & $\begin{array}{l}\text { Compliant } \\
(n=655)\end{array}$ & $\begin{array}{l}\text { Noncompliant* } \\
(n=293)\end{array}$ & $\begin{array}{l}P \text { for } \\
\text { difference }\end{array}$ \\
\hline $\begin{array}{l}\text { Bayesian probability of } \geq 5 \text { days/wk } \\
\geq 30 \mathrm{~min} / \text { day of } 10 \text {-minute bouts of } \\
\text { at least moderate intensity movement, } \\
\% \text { of adults at or exceeding threshold }\end{array}$ & $2.5(1.7-3.3)$ & I.I $(0.3-1.9)$ & 0.056 \\
\hline Lipids profile & & & \\
\hline †Total serum cholesterol (mmol/L) & $5.50(5.39-5.62)$ & $5.48(5.32-5.66)$ & 0.89 \\
\hline †Serum HDL cholesterol (mmol/L) & $1.28(1.25-1.32)$ & $1.24(1.19-1.30)$ & 0.30 \\
\hline †Serum LDL cholesterol (mmol/L) & $3.09(2.96-3.22)$ & $3.20(2.99-3.42)$ & 0.45 \\
\hline †Plasma triglycerides (mmol/L) & $1.71(1.60-1.83)$ & $1.85(1.63-2.09)$ & 0.26 \\
\hline
\end{tabular}

Notes: The comparison on questionnaire-derived measures and lipid profile variables was adjusted for age, gender, race/ethnicity, education attainment, and BMI category. The comparison on accelerometer-derived measures was adjusted for age, gender, race/ethnicity, education attainment, BMI category and days of valid accelerometer wear. *This group included participants who responded "no", "do not know" and refusal. †The variables are log-transformed before the analysis due to right skewness; the values shown have been transformed back to original scales.

Abbreviations: BMI, body mass index; Cts, counts; HDL, high-density lipoprotein; LDL, low-density lipoprotein; MET, metabolic equivalent; MVPA, moderate to vigorous physical activity; PAQ, physical activity questionnaire.

cholesterol-lowering medication did not change the results significantly.

\section{Discussion}

Physician advice has been shown to be effective in changing people's lifestyle behaviors including physical activity, ${ }^{18-23,25,26}$ whereas other studies showed that physician advice on increasing physical activity does not seem to be effective in changing behavior. ${ }^{27,28}$ Nonetheless, physician advice, counseling, and follow-up are important components of the social-environmental supports needed to increase population physical activity levels. In this study, about two thirds of the individuals with elevated blood cholesterol recalled being given the advice from health professionals to increase physical activity to control their condition. Among these individuals, our analyses suggest that members of this group tended to heed this advice.

Our findings showed that people who reported to have increased their physical activity levels either by following physician's advice or on their own might have done so as reflected in physical activity levels measured by both questionnaire and accelerometer. Group differences were observed in the majority of measures of physical activity type (ie, cardio, strengthening, flexibility, moderate-intensity physical activity, vigorous-intensity physical activity) assessed by PAQ. The relevant group differences determined by PAQ were corroborated by the accelerometer findings. The compliance groups differed by all of the accelerometerassessed measures of intensity, duration, and frequency, but not the measure of compliance with physical activity recommendations.
The findings in this report are subject to some limitations. First, the health condition screening, physician's counseling, change in exercise, and physical activity questionnaire data were self-reported, and therefore, subject to biases from misunderstanding the questions, recall, and social desirability. Moreover, self-reported duration of physical activity may include minutes of activity and rest periods. ${ }^{29}$ However, the accelerometer data were not subject to these biases. ${ }^{30}$ Second, although seven days of monitoring has been shown to provide a reasonable representation of usual physical activity, ${ }^{31}$ only one quarter of the NHANES sample wore the instruments for $\geq 10$ hours/day for seven days. ${ }^{30}$ Participants who were excluded because of missing accelerometer data are somewhat different in categorical distributions of age, education, the poverty index and race/ethnicity. ${ }^{17}$ Third, the persons who said they followed their physician's advice to increase physical activity actually had more valid wear days and more wear minutes in valid days; this might partially contribute to the detected difference although valid days of accelerometer wear was controlled in the comparison. Fourth, although gardening and yard work are among the most common leisure-time physical activities, ${ }^{29}$ they were reported as "other" and recoded in the NHANES 2003-2004, introducing the potential for underreporting. Fifth, the waist-mounted, uniaxial accelerometers miss some physical activity that involves upper-body movement (eg, weightlifting, cycling) and load carrying, and all waterborne activity. ${ }^{30}$ However, only $2 \%$ of US adults engage in weightlifting and $1 \%$ engage in cycling or water sports on any given day. ${ }^{29}$ Sixth, accelerometer data analyses are sensitive to the cut-off points that are used to classify minutes 
Table 2 Differences in physical activity (PA) measures (adjusted mean [ $95 \% \mathrm{Cl}]$ ) assessed by PAQ and by accelerometer and lipids profile in nonhypercholesterolemic adults between categories of efforts to increase PA to lower their blood cholesterol

\begin{tabular}{|c|c|c|c|}
\hline Questionnaire-derived measures & $\begin{array}{l}\text { Increased PA on own } \\
\text { effort }(n=552)\end{array}$ & $\begin{array}{l}\text { Did not increase* PA } \\
(n=2644)\end{array}$ & $\begin{array}{l}P \text { for } \\
\text { difference }\end{array}$ \\
\hline \multicolumn{4}{|l|}{$\begin{array}{l}\text { I) PA based on different domains } \\
\text { (number of sessions per month) }\end{array}$} \\
\hline Exercise & $25.9(22.4-29.4)$ & $12.9(\mid 1.3-14.5)$ & $<0.0001$ \\
\hline Sports & $2.6(1.6-3.6)$ & $1.5(1.3-1.7)$ & 0.02 \\
\hline Recreation & $1.9(1.3-2.5)$ & $1.4(1.2-1.6)$ & 0.05 \\
\hline Household PA & $7.4(6.2-8.6)$ & $6.8(6.0-7.6)$ & 0.33 \\
\hline Transportation PA & $4.6(1.5-7.7)$ & $3.5(2.3-4.7)$ & 0.37 \\
\hline All PA & $42.5(36.5-48.2)$ & $26.0(23.6-28.3)$ & $<0.0001$ \\
\hline \multicolumn{4}{|l|}{$\begin{array}{l}\text { 2) PA based on different } \\
\text { domains (total MET-minutes) }\end{array}$} \\
\hline Exercise & $4389(3657-5|2|)$ & 2276 ( $1974-2577)$ & $<0.0001$ \\
\hline Sports & |26| (876-I,646) & $882(7 \mid 6-1,049)$ & 0.04 \\
\hline Recreation & 657 (419-895) & $680(529-830)$ & 0.86 \\
\hline Household PA & $2594(210 \mathrm{I}-3086)$ & 2584 (2449-27।8) & 0.38 \\
\hline Transportation PA & 503 (297-708) & $40 \mathrm{I}(267-536)$ & 0.38 \\
\hline All PA & $9440(8170-10710)$ & $6822(6179-7465)$ & 0.0004 \\
\hline \multicolumn{4}{|l|}{ 3) Type of PA: } \\
\hline \multicolumn{4}{|l|}{ Number of sessions per month } \\
\hline Cardio & $37.9(32.4-43.4)$ & $23.9(21.7-26.0)$ & $<0.0001$ \\
\hline Strengthening & $2.2(1.6-2.8)$ & $0.9(0.7-1.1)$ & 0.0003 \\
\hline Flexibility & $2.5(1.3-3.7)$ & I.3 (0.9-I.7) & 0.046 \\
\hline \multicolumn{4}{|l|}{ Total MET-minutes } \\
\hline Cardio & 9038 (7769-10307) & $6634(6012-7255)$ & 0.0008 \\
\hline Strengthening & $288(191-385)$ & $122(9 \mid-153)$ & 0.003 \\
\hline Flexibility & $114(67-160)$ & $67(47-86)$ & 0.09 \\
\hline \multicolumn{4}{|l|}{ 4) PA based on intensity } \\
\hline \multicolumn{4}{|l|}{ Number of sessions per month } \\
\hline Moderate intensity PA & $26.7(22.4-31.0)$ & $19.2(|7.2-2| .2)$ & 0.0003 \\
\hline Vigorous intensity & $11.1(9.1-13.1)$ & $4.7(3.9-5.5)$ & $<0.0001$ \\
\hline \multicolumn{4}{|l|}{ Total MET-minutes } \\
\hline Moderate intensity PA & $5453(4630-6246)$ & 4851 (4338-5363) & 0.13 \\
\hline Vigorous intensity & $3585(2890-4280)$ & I 783 (1639-2075) & $<0.0001$ \\
\hline Accelerometer-derived measures & $N=460$ & $N=2,009$ & \\
\hline $\begin{array}{l}{ }^{\dagger} \text { Mean intensity counts/minute on wear } \\
\text { periods from all valid days }\end{array}$ & $286(270-304)$ & $272(262-283)$ & 0.07 \\
\hline $\begin{array}{l}{ }^{\dagger} \text { Mean counts/minute of most intense } \\
10 \mathrm{~min} / \text { week }\end{array}$ & 275I (2543-2975) & 2489 (2393-2588) & 0.044 \\
\hline $\begin{array}{l}\text { †Mean duration (minutes) of moderate } \\
\text { and vigorous activity bouts (minimum } \\
\text { I minute bouts) per day from all valid days }\end{array}$ & $13.4(\mid 1.6-15.6)$ & $11.7(10.5-13.0)$ & 0.03 \\
\hline $\begin{array}{l}\text { †Mean duration (minutes) of } \\
\text { moderate and vigorous activity bouts } \\
\text { ( } 8 \text { out of } 10 \text { minute bouts) per day } \\
\text { from all valid days }\end{array}$ & $2.0(1.5-2.7)$ & $1.5(1.2-1.8)$ & 0.046 \\
\hline $\begin{array}{l}\text { Days/week } \geq 10 \mathrm{~min} \text { of MVPA bouts } \\
\text { ( } 8 \text { out of } 10 \text { minutes) from all valid days }\end{array}$ & I.I (0.9-I.3) & $0.9(0.8-1.0)$ & 0.026 \\
\hline
\end{tabular}


Table 2 (Continued)

\begin{tabular}{|c|c|c|c|}
\hline Questionnaire-derived measures & $\begin{array}{l}\text { Increased PA on own } \\
\text { effort }(n=552)\end{array}$ & $\begin{array}{l}\text { Did not increase* PA } \\
(n=2644)\end{array}$ & $\begin{array}{l}P \text { for } \\
\text { difference }\end{array}$ \\
\hline $\begin{array}{l}\text { Bayesian probability of } \geq 5 \text { days/wk } \\
\geq 30 \mathrm{~min} / \text { day of } 10 \text {-minute bouts of at } \\
\text { least moderate intensity movement, } \% \\
\text { adults at or exceeding threshold } \\
\text { Lipids profile }\end{array}$ & $4.0(2.7-5.2)$ & $3.1(2.5-3.7)$ & 0.21 \\
\hline †Total serum cholesterol (mmol/L) & $4.93(4.85-5.01)$ & $4.95(4.91-4.99)$ & 0.73 \\
\hline †Serum HDL cholesterol (mmol/L) & $1.35(1.31-1.38)$ & $1.35(1.33-1.38)$ & 0.81 \\
\hline †Serum LDL cholesterol (mmol/L) & $2.78(2.67-2.89)$ & $2.79(2.73-2.85)$ & 0.85 \\
\hline †Plasma triglycerides $(\mathrm{mmol} / \mathrm{L})$ & $1.24(1.12-1.37)$ & $1.26(1.20-1.32)$ & 0.82 \\
\hline
\end{tabular}

Notes: The comparison on questionnaire-derived measures was adjusted for age, gender, race/ethnicity, education attainment and BMI category. The comparison on accelerometer-derived measures was adjusted for age, gender, race/ethnicity, education attainment, BMI category and days of valid accelerometer wear. *This group included participants who responded "no", "do not know" and refusal. †The variables are log-transformed before the analysis due to right skewness; the values shown have been transformed back to original scales.

Abbreviations: BMI, body mass index; Cts, counts; HDL, high-density lipoprotein; LDL, low-density lipoprotein; MET, metabolic equivalent; MVPA, moderate to vigorous physical activity; PAQ, physical activity questionnaire.

as light or moderate-to-vigorous intensity. ${ }^{30}$ We selected cut off-points based on walking and treadmill studies that have been published for other analyses of this NHANES data ${ }^{17,30}$ in an attempt to discriminate volitional exercise from other activities. Finally, this is a cross-sectional study. Whether a person actually increased their activity level over time is not certain because we did not perform any baseline physical activity measurement. For the same reason, we are uncertain whether the finding of virtually no significant difference in lipid profiles between participants who increased physical activity levels and those who did not is because the increased physical activity is not significant enough to produce any beneficial effects in changing lipid profiles.

Despite low activity levels in hypercholesterolemic adults, there has been a drop in the percentage of people with high cholesterol and the levels of the average blood cholesterol over the last few decades. ${ }^{32}$ This trend might be largely attributed to the increase in the use of cholesterollowering medication. ${ }^{33-35}$ The decrease in the prevalence of hypercholesterolemia and average cholesterol levels would be presumably deepened if hypercholesterolemic or nonhypercholesterolemic persons adopt or maintain healthy lifestyles. Nonetheless, this population-based study showed that individuals who reported having increased their physical activity levels to control blood cholesterol do not possess more favorable lipid profiles than their counterparts, no matter whether they were told to have high cholesterol or not. Obviously, given the prevalent use of cholesterol-lowering medication, the "dosage" of increased physical activity may not be sufficient enough to bring out any significant difference in the outcome at the population level.
There are very few studies that co-relate self-reported measures with direct measurement of physical activity using an accelerometer. ${ }^{19}$ More such studies are needed to examine the effect of lifestyle modification such as increasing physical activity on improving blood cholesterol or other subclinical conditions.

In conclusion, our results suggest that persons who reported following physician advice to increase physical activity to increase physical activity are likely to have done so. Moreover, self report of physical activity to control cholesterol appears to be valid. Results suggest that self reports might be valid to track progress in lifestyle modifications towards cholesterol control. However, more intensive interventions are called for to increase physical activity in the communities in order to achieve significant health benefits including lower cholesterol levels in US adults.

\section{Acknowledgments}

The findings and conclusions in this article are those of the authors and do not necessarily represent the position of the Centers for Disease Control and Prevention. The authors report no conflicts of interest in this work.

\section{References}

1. Expert Panel on Detection, Evaluation, and Treatment of High Blood Cholesterol in Adults. Executive Summary of The Third Report of The National Cholesterol Education Program (NCEP) Expert Panel on Detection, Evaluation, And Treatment of High Blood Cholesterol In Adults (Adult Treatment Panel III). JAMA. 2001;285:2486-2497.

2. Ross R. Atherosclerosis is an inflammatory disease. Am Heart $J$. 1999; 138:S419.

3. Abbott RD, Wilson PW, Kannel WB, Castelli WP. High density lipoprotein cholesterol, total cholesterol screening, and myocardial infarction. The Framingham Study. Arteriosclerosis. 1988;8:207-211. 
4. Assmann G, Schulte H. Relation of high-density lipoprotein cholesterol and triglycerides to incidence of atherosclerotic coronary artery disease (the PROCAM experience). Prospective Cardiovascular Munster study. Am J Cardiol. 1992;70:733-737.

5. Gordon DJ, Probstfield JL, Garrison RJ, et al. High-density lipoprotein cholesterol and cardiovascular disease. Four prospective American studies. Circulation. 1989;79:8-15.

6. Wilson PW, Abbott RD, Castelli WP. High density lipoprotein cholesterol and mortality. The Framingham Heart Study. Arteriosclerosis. 1988;8:737-741.

7. Mackness MI, Arrol S, Abbott C, Durrington PN. Protection of lowdensity lipoprotein against oxidative modification by high-density lipoprotein associated paraoxonase. Atherosclerosis. 1993;104:129-135.

8. Ballantyne FC, Clark RS, Simpson HS, Ballantyne D. The effect of moderate physical exercise on the plasma lipoprotein subfractions of male survivors of myocardial infarction. Circulation. 1982;65:913-918.

9. Brownell KD, Bachorik PS, Ayerle RS. Changes in plasma lipid and lipoprotein levels in men and women after a program of moderate exercise. Circulation. 1982;65:477-484.

10. Couillard C, Despres JP, Lamarche B, et al. Effects of endurance exercise training on plasma HDL cholesterol levels depend on levels of triglycerides: evidence from men of the Health, Risk Factors, Exercise Training and Genetics (HERITAGE) Family Study. Arterioscler Thromb Vasc Biol. 2001:1226-1232.

11. Durstine JL, Miller W, Farrell S, Sherman WM, Ivy JL. Increases in HDL-cholesterol and the HDL/LDL cholesterol ratio during prolonged endurance exercise. Metabolism. 1983;32:993-997.

12. Gordon DJ, Witztum JL, Hunninghake D, Gates S, Glueck CJ. Habitual physical activity and high-density lipoprotein cholesterol in men with primary hypercholesterolemia. The Lipid Research Clinics Coronary Primary Prevention Trial. Circulation. 1983;67:512-520.

13. Huttunen JK, Lansimies E, Voutilainen E, et al. Effect of moderate physical exercise on serum lipoproteins. A controlled clinical trial with special reference to serum high-density lipoproteins. Circulation. 1979;60:1220-1229.

14. Kokkinos PF, Fernhall B. Physical activity and high density lipoprotein cholesterol levels: What is the relationship? Sports Med. 1999;28: 307-314.

15. US Department of Health and Human Services. Physical Activity Guidelines for Americans. Hyattsville, MD: US Department of Health and Human Services, 2008. Available at http://www.health. gov/paguidelines/guidelines/default.aspx. Accessed on July 10, 2009.

16. Prevalence of self-reported physically active adults - United States, 2007. MMWR Morb Mortal Wkly Rep. 2008;57:1297-1300.

17. Metzger JS, Catellier DJ, Evenson KR, Treuth MS, Rosamond WD, Siega-Riz AM. Patterns of objectively measured physical activity in the United States. Med Sci Sports Exerc. 2008;40:630-638.

18. Bull FC, Jamrozik K. Advice on exercise from a family physician can help sedentary patients to become active. Am J Prev Med. 1998;15:85-94.

19. Calfas KJ, Long BJ, Sallis JF, Wooten WJ, Pratt M, Patrick K. A controlled trial of physician counseling to promote the adoption of physical activity. Prev Med. 1996;25:225-233.

20. Greenlund KJ, Giles WH, Keenan NL, Croft JB, Mensah GA, Huston SL. Physician advice, patient actions, and health-related quality of life in secondary prevention of stroke through diet and exercise. Stroke. 2002;33:565-570.
21. Kreuter MW, Chheda SG, Bull FC. How does physician advice influence patient behavior? Evidence for a priming effect. Arch Fam Med. 2000;9:426.

22. Lewis BS, Lynch WD. The effect of physician advice on exercise behavior. Prev Med. 1993;22:110-121.

23. Wofford TS, Greenlund KJ, Croft JB, Labarthe DR. Diet and physical activity of U.S. adults with heart disease following preventive advice. Prev Med. 2007;45:295-301.

24. Westerterp KR. Physical activity assessment with accelerometers. Int J Obes. 1999;23:S45-S49.

25. Centers for Disease Control and Prevention (CDC). Physician advice and individual behaviors about cardiovascular disease risk reduction - seven states and Puerto Rico, 1997. MMWR Morb Mortal Wkly Rep. 1999;48:74-77.

26. Greenlund KJ, Giles WH, Keenan NL, Croft JB, Mensah GA. Physician advice, patient actions, and health-related quality of life in secondary prevention of stroke through diet and exercise. Stroke. 2002;33: 565-570.

27. Egede LE. Lifestyle modification to improve blood pressure control in individuals with diabetes: is physician advice effective? Diabetes Care. 2003;26:602-607.

28. Kant AK, Miner P. Physician advice about being overweight: association with self-reported weight loss, dietary, and physical activity behaviors of US adolescents in the National Health and Nutrition Examination Survey, 1999-2002. Pediatrics. 2007;119: e142-e147.

29. Ham SA, Kruger J, Tudor-Locke C. Participation by U.S. adults in sports, exercise, and recreational physical activities. J Phys Act Health. 2009;6:6-14.

30. Troiano RP, Berrigan D, Dodd KW, MÂSse LC, Tilert T, McDowell M. Physical activity in the United States measured by accelerometer. Med Sci Sports Exerc. 2008;40:181-188.

31. Matthews CE, Ainsworth BE, Thompson RW, Bassett Jr DR. Sources of variance in daily physical activity levels as measured by an accelerometer. Med Sci Sports Exerc. 2002;34:1376-1381.

32. Ford ES, Mokdad AH, Giles WH, Mensah GA. Serum total cholesterol concentrations and awareness, treatment, and control of hypercholesterolemia among US adults: findings from the National Health and Nutrition Examination Survey, 1999 to 2000. Circulation. 2003;107:2185-2189.

33. Arnett DK, Jacobs Jr DR, Luepker RV, Blackburn H, Armstrong C, Claas SA. Twenty-year trends in serum cholesterol, hypercholesterolemia, and cholesterol medication use: the Minnesota Heart Survey, 1980-1982 to 2000-2002. Circulation. 2005;112: 3884-3891.

34. Lemaitre RN, Furberg CD, Newman AB, et al. Time trends in the use of cholesterol-lowering agents in older adults: the Cardiovascular Health Study. Arch Intern Med. 1998;158:1761-1768.

35. Siegel D, Lopez J, Meier J. Use of cholesterol-lowering medications in the United States from 1991 to 1997. Am J Med. 2000;108: 496-499.

36. National Center for Health Statistics. National Health and Nutrition Examination Survey 2003-2004. Documentation, codebook, and frequencies. SP questionnaire component: physical activity individual activities data. Available from: http://www.cdc.gov/nchs/data/ nhanes/nhanes_03_04/paqiaf_c.pdf. Accessed on July 10, 2009. 


\section{Appendix I}

\section{Physical activity determined by interview}

The participants were asked about their participation in the past 30 days for transportation physical activity, household activity, and moderate- and vigorous-intensity physical activities during leisure time. Transportation activity was defined as walking or bicycling "as part of getting to and from work, or school, or to do errands." Participants were asked to provide the number of times per day, per week, or per month, and the total time per day spent in transportation physical activity. Household activity was described as "tasks in or around your home or yard for at least 10 minutes that required moderate or greater physical effort." To determine frequency and duration, participants were asked to report the number of times in the past 30 days that they participated and the minutes that the activity was performed each time. For leisure-time physical activity, participants were asked to indicate frequency (times/30 days) and duration (minutes/time) of participation in specific activities in bouts of at least 10 minutes. Leisure-time physical activity included sports (16 activities), exercise (14 activities), and recreational activities (14 activities). Gardening and yard work were volunteered as "other" leisure-time activities. Participants self-selected the intensity of each activity as moderate (ie, light sweating, or slight to moderate increase in breathing or heart rate) or vigorous (ie, heavy sweating or large increase in breathing or heart rate) intensity. Strengthening activities were included in the lists of moderate and vigorous physical activity.

Ten sets of physical activity indicators were created from the data by classifying specific physical activity into categories of physical activity domains and subdomains, type, and intensity (see Table S1). Each activity was assigned a metabolic equivalent (MET) value based on the Compendium of Physical Activities..$^{36}$ One metabolic unit (MET) equals $3.5 \mathrm{ml} \mathrm{O} / \mathrm{kg}$ body weight/minute of oxygen expenditure. MET values were used to classify activities as moderate- intensity activities (3.0-6.0 METs) and vigorous-intensity activities ( $>6.0$ METs). For each of the 10 sets of indicators, the number of sessions per month and total MET-minutes/month were computed. The number of sessions per month was the sum of the number of sessions in the past 30 days for all activities in the category. MET-minutes/month were determined by the product of the MET value, number of sessions/month, and minutes/session.
Total MET-minutes/month was the sum over all activities in the category.

\section{Physical activity determined by accelerometer}

The details of physical activity measurement using accelerometer data from NHANES 2003-2004 have been published elsewhere. ${ }^{17,30}$ To objectively monitor independent physical activity, participants were instructed to wear the accelerometer for seven days on the right hip attached by an elastic belt during waking hours, with the exception of time spent bathing and in water-based activities. Accelerometers were programmed to record activity "counts" in one-minute intervals. After the monitoring period, accelerometers were returned by mail to the NHANES contractor, where data was downloaded and the monitors were checked for calibration to determine whether the units were still within the manufacturer's specifications.

Of the 4,637 eligible adults who were asked the survey question about cholesterol screening, 3,652 were in our analytic sample defined as having at least one valid day of accelerometer data (mean $5.3 \pm 1.8$ days; $13.9 \pm 1.8$ hours/day). We excluded those who had accelerometer data but had zero valid days, or those whose monitors were not in calibration upon return. Valid days were defined as having at least 10 hours of monitor wear. Wear time was determined by subtracting nonwear time from 24 hours. Nonwear time was defined as an interval of at least 60 consecutive minutes of zero counts, with allowance for 1-2 minutes of observations of 1-100 counts/minute. Implausible data values that were consistent with monitor malfunction (eg, 32,767 counts/minute) were replaced by imputed values that were the averages of the counts recorded in the minutes immediately preceding and following the implausible value(s).

The following variables were created to assess physical activity volume, intensity, duration, and frequency: 1) total counts/week, 2) mean counts/minute, 3) mean intensity of the most intense 10-minute period during the week, 4) mean minutes/day of at least moderate- intensity movement, 5) mean minutes/day of at least moderate-intensity movement in 10-minute bouts, 6) days of at least one 10-minute bout of at least moderate-intensity movement, 7) accumulation of $\geq 30$ minutes/day of 10-minute bouts of at least moderate intensity movement on $\geq 5$ days. Mean counts/ minute was calculated by dividing the sum of activity counts for a valid day by the number of minutes of wear time in that 
day across all valid days. The mean intensity of the most intense 10-minute period during the week was the maximum value of 10-minute moving averages of activity counts across all days, excluding periods with invalid data.

To determine whether a participant engaged in at least moderate-intensity locomotion in a given minute, the assessment of at least moderate-intensity movement compared the activity counts to a threshold. The threshold 2,020 counts/minute was selected because a weighted average of thresholds from four studies that based criteria on treadmill or track walking estimated that 2,020 counts/minute is equivalent to moderate-intensity (eg, three METs). ${ }^{30}$ Thus, mean minutes per day of at least moderate-intensity movement was the average across all valid days of the sum of all minutes (minimum one minute bouts) with activity counts exceeding the threshold 2,020 counts/minute.

To compare patterns of locomotion with recommendations for physical activity of at least moderate intensity (moderate to vigorous physical activity, MVPA), eight out of 10-minute bouts were derived from count data. Bouts were defined as 10 or more minutes above the threshold of 2,020 counts/minute, with allowance for interruptions of one or two minutes below the threshold. A bout was terminated by three minutes below the threshold. To estimate the daily duration of physical activity, mean minutes per day of at least moderate-intensity movement in 10-minute bouts was determined by summing the minutes in a day in 10-minute bouts across all valid days. Frequency was calculated by summing the days of at least one 10-minute bout of at least moderate-intensity movement. Finally, compliance with physical activity recommendations at the time of the NHANES study was determined by the accumulation of $\geq 30$ minutes/day of 10-minute bouts of at least moderateintensity movement on $\geq 5$ days per week. A Bayesian indicator of the probability of obtaining $\geq 5$ days per week was used for the analysis. ${ }^{30}$ 
Table SI Classifications of physical activities into sets of indicators using NHANES 2003-2004 data

\section{Indicators}

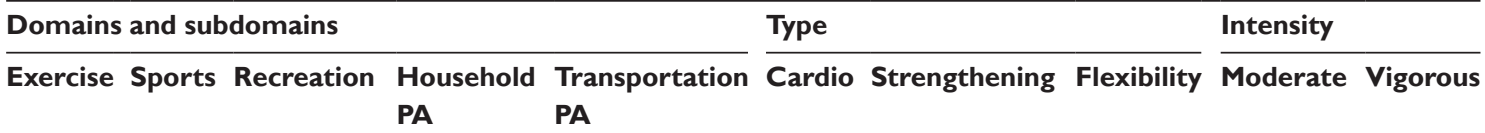

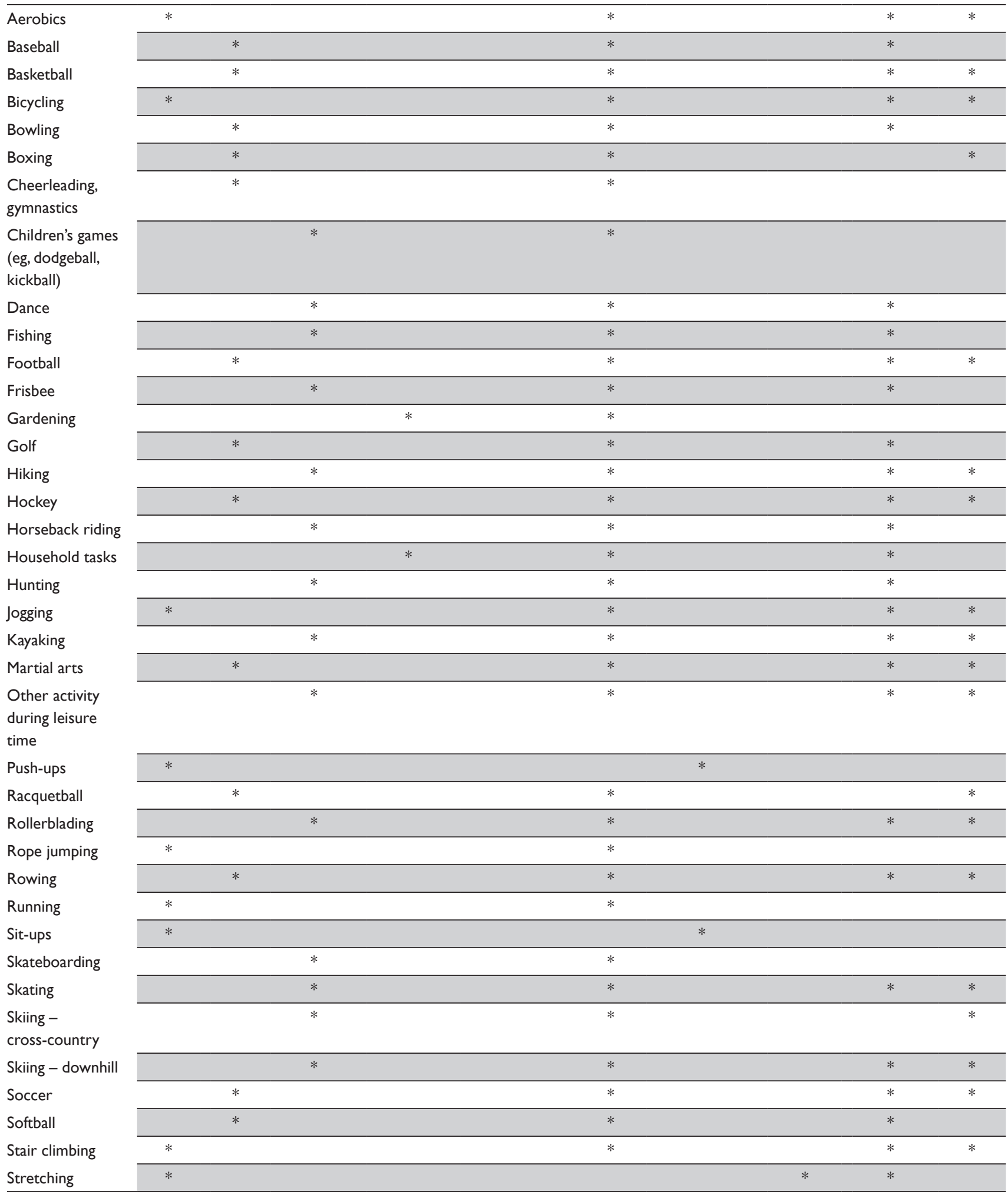

(Continued) 
Table SI (Continued)

Indicators

Domains and subdomains Intensity

Exercise Sports Recreation Household Transportation Cardio Strengthening Flexibility Moderate Vigorous PA

PA

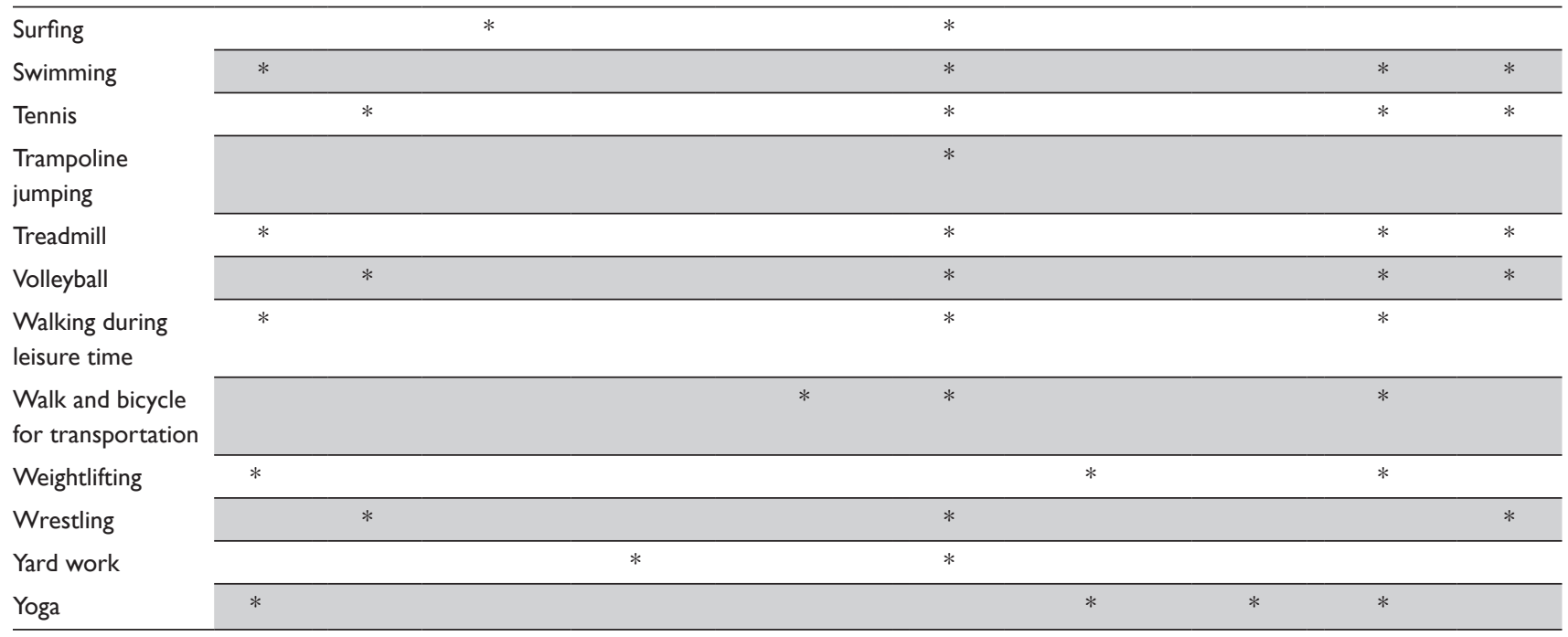

Abbreviation: PA, physical activity.

Vascular Health and Risk Management

\section{Publish your work in this journal}

Vascular Health and Risk Management is an international, peerreviewed journal of therapeutics and risk management, focusing on concise rapid reporting of clinical studies on the processes involved in the maintenance of vascular health; the monitoring, prevention and treatment of vascular disease and its sequelae; and the involvement of

\section{Dove press}

metabolic disorders, particularly diabetes. This journal is indexed on PubMed Central and MedLine. The manuscript management system is completely online and includes a very quick and fair peer-review system, which is all easy to use. Visit http://www.dovepress.com/ testimonials.php to read real quotes from published authors. 